\title{
A Review on Applications of the Wavelet Transform Technique in Spectral Analysis
}

\section{Medhat ME*}

Experimental Nuclear Physics Department, Nuclear Research Centre, Cairo, Egypt

\begin{abstract}
Starting from 1989, a new technique known as wavelet transforms (WT) has been applied successfully for analysis of different types of spectra. WT offers certain advantages over Fourier transforms for analysis of signals. A review of using this technique through spectral analysis is presented. The mathematical principles of applying WT in processing gamma spectra have been discussed.
\end{abstract}

Keywords: Wavelet transform; Wavelet packet transform; Compression; Smoothing; De-noising; Spectral analysis

\section{Introduction}

The wavelet transform WT has attracted recent interest in applied mathematics for signal and image processing [1]. This new mathematical technique has been demonstrated to be fast in computation with localization and quick decay properties in contrast to existing popular methods, especially, the fast Fourier transform FFT. Theory of the WT was developed extensively in 1980s [2]. It may be viewed as a synthesis of ideas originating from engineering sub-band coding), physics (coherent states and renormalization group).

WT became a tool widely used for signal analysis. From 1989 onwards, the WT has been applied for signal processing in spectral studies owing to its efficiency, large number of basis functions available, and high speed in data treatment. One of the main features of WT is that it may decompose a signal directly according to the frequency and represent it in the frequency domain distribution state in the time domain. In the transformation, both time and frequency information of the signal are retained. Another important thing, the time domain can be replaced by other domains such as energies of channel numbers. With proper identification of the scales with frequency, higher frequency signals can be separated from the lower one, in the sense that it has zoom-in and zoom-out capability at any frequency. Since WT can focus on any small part of a signal, it has been called a mathematical microscope [3]. Another feature of WT is that the development of signals in the frequency domain can be constituted with a flexible choice of waveforms rather than with only trigonometric ones as a basis. For instance, in FFT, only the sine and cosine functions can be chosen as the basis.

In contrast, a lot of such functions can be selected in WT. Therefore, WT is a more powerful method to depict relationships among different variables.

In analytical chemistry, applications of WT can be found in the following areas: flow injection analysis FIA, high performance liquid chromatography HPLC, infrared IR spectrometry, mass spectrometry MS, nuclear magnetic resonance NMR spectrometry, ultravioletvisible UV-VIS spectrometry and voltammetry. Besides, WT has also been employed in studying quantum chemistry and chemical physics.

Readers who are interested in learning more general theory of wavelets might consult Refs. [4-8]. A number of public domain wavelet transforms software, which can be downloaded from the Internet, are available for non-commercial, research and educational users. Readers can find more information on this software via the Internet or Refs. [9-14].

Many wavelet functions have been proposed by various workers. The simplest one, the Haar wavelet, which is also the first member of the family of Daubechies wavelets, has been known for more than 80 years in various mathematical fields. In addition, there are many other wavelet families such as the Meyer wavelet, Coiflet wavelet, spline wavelet, orthogonal wavelet and local cosine basis and others. Beside the Daubechies wavelet, the spline wavelet is another type of wavelet function that has been used chemical studies [15-20].

Coifman and Wickerhauser introduced the concept of the wavelet packet transform WPT for signal processing to generalize the timefrequency analysis of WT [21].

In the discrete wavelet transform DWT treatment, only outputs from the low-pass filter are processed by WT. However, in the WPT treatment, both outputs from the low-pass and high-pass filters are manipulated by WT. The wavelet packet is a family of basis functions which allow one to select different orthogonal bases for a given signal vector with finite energies. The standard wavelet basis is an orthogonal basis among the family and each basis is called a wavelet packet basis. Different effectiveness of concentrating energy of a given signal may be attained with different distinct wavelet packet bases chosen.

Coifman et al. [22] proposed a selection scheme for the best wavelet packet basis with certain selection criteria such as the Shannon-Weaver entropy measure function.

In a recent, paper Walczak and Massart [23] developed a new method, which is based on the variance spectrum, for best-basis selection with WPT of a set of signals. They explained that the existing best-basis selection methods such as the Shannon-Weaver entropy method are only good for compression and de-noising of individual signals but not for a set of signals. By adopting their method, a single

${ }^{*}$ Corresponding author: Medhat ME, Experimental Nuclear Physics Department Nuclear Research Centre, P.O.13759, Cairo, Egypt, Tel: +86 13693344534; E-mail: medhatme@ymail.com

Received April 25, 2015; Accepted June 05, 2015; Published June 11, 2015

Citation: Medhat ME (2015) A Review on Applications of the Wavelet Transform Technique in Spectral Analysis. J Appl Computat Math 4: 224. doi:10.4172/2168 9679.1000224

Copyright: @ 2015 Medhat ME. This is an open-access article distributed under the terms of the Creative Commons Attribution License, which permits unrestricted use, distribution, and reproduction in any medium, provided the original author and source are credited. 
best basis will be chosen for the whole set of data rather than different best-base for an individual signal in a data set.

Recently, Walczak and Massart [24] published a tutorial on the introduction of WPT for noise suppression and signal compression. They also published another paper on the introduction of WT in analytical chemistry [25]. The basic principles of WPT calculation and best-basis selection were introduced in their paper.

In addition, Alsberg et al. [26] presented another tutorial on the introduction of WT to chemometricians. The basic principles and the properties of continuous and discrete wavelet transforms and WPT were mentioned in their tutorial. These workers pointed out five major applications of WT in chemistry including signal de-noising, baseline removal, zero crossing, signal compression and wavelet regression.

Wang et al. [27] also published a paper on the introduction of WT and its applications in chemistry in China. They pointed out another five major applications of WT that include spectral data compression, modification of the quality of chemical signals de-noising, quantum chemistry calculation, chemical dynamic analysis, and chemical fractals.

Depczynski et al. [28] published a paper that introduced the mathematical background of the fast wavelet transform FWT on compact intervals. They emphasized on the problem of periodization of a general signal that leads to instabilities near the boundary of the interval.

\section{Brief Description of Wavelet Transform}

A wavelet transform involves the decomposition of a signal $\mathrm{f}(\mathrm{x})$ at different scales and positions. In a WT treatment, all basis functions are $\Psi_{a, b}(x)$ derived from a mother wavelet $\Psi(x)$ through the following dilation and translation processes:

$$
\Psi_{a, b}(x)=a^{-1 / 2} \Psi\left(\frac{x-b}{a}\right) \quad \mathrm{a}, \mathrm{b} \in \mathrm{R}, \mathrm{a} \neq 0
$$

where $\mathrm{a}$ and $\mathrm{b}$ are, respectively, the scale and position parameters, with $\mathrm{a}>0$ and $\mathrm{b}$ having arbitrary values. The continuous wavelet transform of $f(x)$ is given by:

$$
W_{\Psi}(a, b) f=\frac{1}{\sqrt{C_{\Psi}}} \int_{-\infty}^{\infty} \Psi_{a, b}(x) f(x) \mathrm{d} x,
$$

where $\mathrm{C}_{\Psi}$ is the normalization constant.

In practical computation, since the spectrum to be analyzed is often discrete sampling data, the discrete form of Eq. (2) is necessarily used, which can be expressed as:

$$
W_{\Psi}(a, b) f=\frac{1}{N} \frac{1}{\sqrt{|a|}} \sum_{k=1}^{N} \frac{1}{\sqrt{C_{\Psi}}} \Psi\left(\frac{x-b}{a}\right) f(x)
$$

The formula (3) does not contain integral and this fact allows one to apply WT method for analyzing spectrum.

The mother wavelet $\Psi(x)$ is chosen to serve as a prototype for all basis function in the process. All basis function that used are the dilated (or compressed) and shifted versions of the mother wavelet. There are a number of functions that are used for this purpose.

Since gamma spectra are like Gaussian shape it will be good choice if the mother wavelet from the family of gauss functions. Marr wavelet or the Mexican hat is expressed as:

$$
\Psi(x)=\left(1-x^{2}\right) e^{-x^{2} / 2}
$$

Marr wavelet is the second derivative of gauss function. Schematic diagram of Marr wavelet is shown in Figure 1.

\section{Signal Smoothing and De-noising}

One of the main goals in spectral analysis is to extract useful information from recorded data. However, the achievement of this goal is usually complicated by the presence of noise ( e.g. background detected with the signal ). Nowadays, most of detected instruments are controlled by computers. It has become a common practice to reduce the noise by employing digital processing methods such as filtering. In the past decades, a large number of filters have been developed in different fields of science and technology. In spite of the existence of diverse filters, only a few, such as Savitzky-Golay, Fourier and Kalman filters $[29,30]$, are extensively used by analyst. The most common way of noise suppression is by applying a suitable filter to the raw data. Noise is present in spectral data in the form of a high frequency signal and causes singularities in data treatment. The two most important properties of WT are that (1) singularities of a signal can be detected by the WT maxima (9) and (2) signal frequencies can be separated at different scales.

A tutorial on noise suppression with WPT was published by Walczak and Massart. They introduced the universal thresholding algorithm as proposed by Donoho [31,32] for selection of coefficients.

Permann and Teitelbaum [33] developed a wavelet fast Fourier transform WFFT to de-noise the milli-volt signal for a transient oscillating chemical reaction.

The Daubechies wavelet function was applied to the signal and the noise components were resolved by FFT on selected parts of the wavelet coefficient coupled with the threshold method. After data processing, the signal was reconstructed to the original domain.

Recently, Mittermayr et al. [34] performed a comparative study in de-noising the Gaussian peak with the use of wavelet, Fourier and Savitzky-Golay filters.

The Gaussian function was chosen in their work because it is commonly used to represent peaks and bands in chromatographic and spectroscopic studies. They invoked WT to simulate data generated

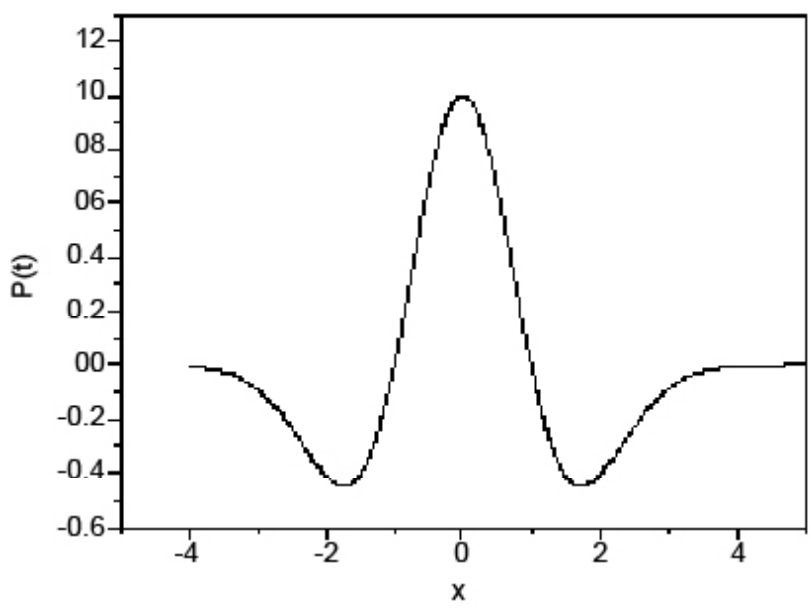

Figure 1: Schematic diagram of Marr wavelet. 
from Gaussian peaks with noise to yield noisy coefficients in the wavelet domain. Then, the so-called universal soft thresholding was applied to these coefficients to give the estimated wavelet coefficients.

Finally, the inverse WT was employed to reconstruct the smoothed data. The proposed wavelet filter can remove noises without distorting the signal peaks. In WT, frequency information is contained in different scales of the multiscale WT representation. The high frequency information resides in the lower resolution levels and low frequency one in the higher levels. Therefore, the effectiveness of the optimal denoising methods depends on the magnitudes of the noise level present and the original signal of interest. The work of Mittermayr et al. [34] shows that under the chosen conditions, the proposed wavelet method gave superior performance over the classical filter techniques in most cases.

Barclay et al. [35] also performed a similar comparative study in de-noising and smoothing of Gaussian peak using wavelet, Fourier and Savitzky-Golay filters. They classified signal de-noising and smoothing as two different processes. Smoothing removes high frequency components of the transformed signal regardless of their amplitudes, whereas de-noising removes small amplitude components of the transformed signal regardless of their frequencies. Their result indicated that DWT de-noising gave better performance than DWT smoothing and DWT smoothing had a drawback. Such drawback was apparent when the peak width of the fine structure was in the order of the Nyquist frequency.

\section{Signal Compression}

Microcomputer systems are widely used nowadays to acquire and store digital spectral data. Computerized databases and spectral libraries are valuable in identification of sample spectra. In general, the more data that is available in a library, the better are the search results. However, as the size of a library increases, more space and longer time are required to store the spectra and search the library. The advancement in microelectronics has greatly enhanced mass storage capacity and processing speed. Archive of information of full spectra becomes more feasible. However, the demand of huge storage capacity is still somewhat inhibited for high-resolution spectra. Even if this problem can be resolved, the computer processing speed and the bandwidth of the phone line or network is still a limiting factor. For instance, in order to transfer a spectral data set to a remote analytical site via phone line or Internet with low bandwidth, data compression is necessary to shorten the transfer time. On the other hand, searching by using the direct search method through a large spectral library is a very lengthy and impractical task.

To speed up the process, researchers may use several approaches. One of them is to reduce the resolution of the spectra to be archived in the spectral domain. A one-fold decrease in the resolution of a spectrum leads to one half of the computational time and space being needed. The second method to speed up data processing is to compress the spectra to give smaller data set. The most commonly used compression technique in spectral studies is the Fourier transform or its variants. The advantages of FT are frequency localization, orthogonality, and the availability of fast numerical algorithms. This technique has been applied extensively in processing digital data. Some workers $[36,37]$ have pointed out that in addition to noise removal, FT can also be used for data compression.

For example, Chau and Tam [38] applied fast FT to minimize the storage of UV-VIS absorption spectra collected from a photodiode array spectrophotometer. In recent years, researchers proposed to make use of DWT or FWT and WPT for data compression [39,40,41]. The mathematical treatment for data compression via WT is similar to that for de-noising.

Data from spectral analysis are transformed to the wavelet domain by employing either FWT or WPT. Then, a thresholding method is employed to select suitable coefficients in the wavelet domain for storage. By utilizing inverse FWT or WPT treatment, the compressed coefficients can be converted back to its original domain.

\section{Applications of the Wavelet Transform in Spectral Analysis}

\section{Neutron activation analysis NAA}

Neutron Activation Analysis NAA is one of the most powerful nondestructive techniques available today for probing elemental analysis of matter. This technique depends on the analysis of gamma spectra GS of the irradiated samples. Only a few publications have been related to the application of WT in GS.

WT methods for smoothing the spectrum by removing the high frequency components in the transform and thereby aiding in finding peaks were discussed by Thakur [42]. The gamma spectrum can be described in the usual energy representation ("Energy domain") by directly samples values of the quantity $h$ (counts) as a function of energy E, e.g. h (E) in energy units (or equivalent channel number). The same data can also be represented as a function of frequency $F$, i.e. $\mathrm{H}(\mathrm{F})$. The "energy domain" and "frequency domain" representations are related by FT.

Press et al. [43] used to analyze spectral data to obtain information of particular $\gamma$-rays to the continuum in the energy spectrum. Because the continuum is characterized by a broad spread of energies even through the original $\gamma$-ray energy was well defined, the transform to frequency space concentrates this contribution from the continuum that spread over many channels and hard to differentiate from noise in the energy spectrum.

\section{Flow injection analysis FIA}

Bos and Hoogendam [44] proposed to use WT to minimize the effect of noise and baseline drift in flow-injection analysis. Usually, peak overlapping in FIA is not a problem because it can be easily avoided by adjusting the sample rate. However, as the FIA system is operated near the detection limits, it is difficult to locate peaks and find the right baseline correction method. It is because weak signals are embedded in the stochastic noise, which is a general problem in FIA.

In Bos and Hoogendam's study, the Morlet wavelet function was utilized to transform the FIA signal into a two-dimensional timefrequency form with both time and frequency information of the signal being retained. After WT treatment, the peak intensity was filtered from noise optimally. The maximum peak position can be searched from the coefficient in the wavelet domain of a well-defined peak as obtained for a sample with a relatively high concentration. The position of this maximum on the horizontal axis of the transform conveys the positional information of the peak whereas its position on the vertical axis gives the noise filtering characteristic that can be obtained.

\section{Chromatography}

Nine papers have been reported on adopting WT in chromatographic data processing so far. Pan et al. [45] applied WT to correct baseline for analysis of rare earth elements in China. 
Shao et al. [46-49], proposed to use WT in quantitative determination of constituting components in overlapping chromatographic peaks. For unresolved chromatograms, different factor analysis methods such as evolving factor analysis [50,51], fixed moving windows evolving factor analysis [52] and heuristic evolving latent projections [53,54] have been reported for resolving overlapping chromatographic peaks. WT is proposed as a novel method to retrieve separate signals from overlapping chromatographic peaks. In Shao's study, the Haar wavelet function was employed to decompose the chromatographic data into localized contributions and to resolve overlapping chromatographic peaks with two and three components, respectively.

WT was proposed for de-noising and baseline correction, determination of factor number, and resolving multi-component overlapping chromatograms. Mittermayr et al. [55] report a work on choosing WT as a tool to improve calibration and detection limit on data from gas chromatography coupled with a microwave induced plasma detector system.

Mittermayr [56] also published a dissertation to discuss the application of WT to chromatographic data processing. His work was mainly focused on the noise structure and smoothing chromatograms.

Shen et al. $[57,58]$ developed a new method which utilized WT to remove the influence of complex backgrounds-spectral background and chromatographic drift, in a HPLC-diode array detector DAD system. The existence of such complex backgrounds would introduce extra factors in the factor analysis calculation as proposed by Maeder and Zilian [59] and Gemperline [60].

\section{Infrared spectroscopy}

Infrared IR spectroscopy has found widespreaduse in identification and characterization of chemicals. Applications of WT in IR spectrometry were reported in nine papers. The first one that employed WT in IR spectroscopy was published by Stark et al. [61]. With the aid of WT, they could roughly separate the mineralogical information in the FT-IR absorbance spectrum from noise and the other signals such as absorbance from adsorbed water and organics. These workers also developed an empirical affine minimaxestimators method to estimate the mass fraction of a given mineral in a mixture using the wavelet coefficients.

Bos and Vrielink [62] published the second paper concerning about identification of mono- and di-substituted benzenes utilizing WT in IR spectrometry.

Application of WPT in IR spectrometry could be found in the works by Walczak et al. [63] and Alsberg et al. [64] .Walczak's research group chose WPT as a tool for improving pattern recognition based on NIR spectra. Haar wavelet and Coifman's best-basis algorithm were used to construct the full WPT decomposition framework for each NIR spectrum.

\section{Mass spectrometry}

In mass spectrometric MS studies, WT were mainly applied in two areas: instrumentation design and secondary ion mass spectrometry SIMS.

Shew [65] in invented a new procedure to determine the relative ion abundances in ion cyclotron resonance mass spectrometry by utilizing WT to isolate the intensity of a particular ion frequency as a function of position or time within the transient ion cyclotron resonance signal. The WT intensity corresponding to the frequency of each ion species as a function of time can be fitted by an exponential decay curve. When extrapolating these curves back in time to the end of the excitation phase, accurate values of the relative abundances of different ions within a sample can be determined. An ion cyclotron resonance mass spectrometer with a Haar wavelet analysis module was set up by Shew. The result of the work indicated that WT can provide high efficiency isolation of individual frequencies in the received signal corresponding to individual species.

Four papers were published by Nikolov et al. [66], Hutter et al. [67] and Wolkenstein et al. [68,69] in the Vienna University of Technology, for wavelet de-noising of secondary ion mass spectroscopy (SIMS) images. SIMS is a type of surface technique for (1) trace analysis, (2) determination of elemental composition, (3) the identity and concentrations of adsorbed species and (4) elemental composition as a function of depth [70]. This surface technique is capable of measuring the distribution of elements with a lateral resolution of $0.1 \mathrm{~mm}$ in the scanning mode. The two-dimensional element distributions generated by scanning SIMS are characterized by Poisson statistics with small integer values. Poor signal statistics is one of the major problems encountered in SIMS measurement especially in trace analysis. As a result, quantification and image processing methods such as classification and edge detection are difficult to apply. Nikolov et al. [66] reported an application of a wavelet shrinkage algorithm for denoising SIMS images following the Poisson distribution.

\section{Nuclear magnetic resonance spectroscopy}

Nuclear magnetic resonance NMR spectroscopy is one of the most powerful non-destructive techniques available today for probing structure of matter. Only a few publications have been related to the application of WT in NMR spectroscopy [71]. In 1989, Guillemain et al. [72] were the first research group to propose an application of WT in NMR spectroscopy. In their work, they aimed at investigating how an appropriate use of WT could lead to an excellent estimation of the frequency of spectral lines in a signal and provide direct information on time domain features of these lines in NMR spectra. These authors reported seven applications of WT in NMR spectroscopy that included estimation of frequency and amplitude modulation laws in both simple and general cases, spectral line subtraction and re-synthesis, ridge extraction, sum of two sine waves and three exponentially decreasing sine waves.

Recently, Neue [73] published another paper on an application of WT in dynamic NMR spectroscopy, which could simplify the analysis of the free induction decay FID signal. Dynamic NMR spectroscopy is a technique used to measure rate parameters for a molecule [74]. The measured resonance frequencies represent the spatial coordinates of spins. Any motion such as bond rotation and other molecular gymnastics may change these frequencies as a function of time. The localization property of WT gives a better picture of the nature of the underlying dynamical process in both the frequency and time domains. The third-order Battle-Lemarie wavelet was employed for determining crystal rotation and first-order kinetics by NMR spectroscopy in their study. They concluded that WT would become a routine method in NMR spectroscopy for data analysis.

\section{Ultraviolet-visible spectroscopy}

Ultraviolet-visible UV-VIS spectroscopy has been used extensively in physical and biological sciences for characterization, identification and quantification of substances [75]. Two papers were published by Chau et al. [39] and Gao et al. [76] on compression and de-noising UV-VIS spectra with WT. 
Citation: Medhat ME (2015) A Review on Applications of the Wavelet Transform Technique in Spectral Analysis. J Appl Computat Math 4: 224. doi:10.4172/2168-9679.1000224

Page 5 of 6

Recently, a novel application of WT was deveoped by Liu et al. [77]. A wavelet neural network was set up and applied to recognize the UVVIS spectra. The Morlet wavelet and line search conjugate gradient optimization method were used in their neural network. The results indicated that the wavelet neural network had a very good recognition power to differentiate minor differences between similar UV-VIS spectra.

\section{Voltammetry}

The wavelet transform has been applied successfully to voltammetric data. Voltammetry is a popular technique in all fields of chemistry used to study redox reactions. Yan and Mo [78] were the first to introduce WT in processing signals from voltammetry. They developed a real time continuous wavelet filter for voltammetric signal processing.

Chen et al. [79] utilized wavelet function for the treatment voltammetric DPV data. In DPV quantitative analysis, it is very difficult to measure the peak height in a sample with low concentration. As a result, it affects the linear detection range of the DPV system. So, these workers employed the Gaussian difference wavelet function to transform the DPV signal obtained.

Application of WPT to voltammetry was found in recent work by Fang and Chen. They investigated the feature of WPT for white noise which is caused by random and irregular process and developed an adaptive wavelet filter for the selection of the optimum critical level of related frequency band automatically in voltammetry. Their outcomes showed that the adaptive wavelet filter could be applied to a system with interference originating from existing power supply', which is useful for the study of fast electron, transfer process.

\section{Conclusion}

Applications of wavelet transform and wavelet packet transform in various fields were reviewed. WT is mainly used in data compression and noise removal in spectral studies owing to its efficiency, large number of basis functions available and high speed in data treatment. In most cases, the performance of the WT treatment is much better than the Fourier transform. Up to now, one patent, two tutorial papers and more than 70 papers on the application of WT to spectral analysis have been published. Signal processing of spectral data via wavelet transform is still under development and the mathematical technique is expected to be one of the most popular methods in the future.

\section{References}

1. Yan-Fang S (2013) A review on the applications of wavelet transform in hydrology time series analysis. Atmospheric Research 122: 8-15.

2. Li B, Chen $X(2014)$ Wavelet-based numerical analysis: $A$ review and classification. Finite Elements in Analysis and Design 81: 14-31.

3. Daubechies I (1988) Orthonormal bases of compactly supported wavelets. Commun. Pure Appl. Math 41: 909-996.

4. Chui CK (1992) An Introduction to Wavelets. Academic Press, New York 49

5. Newland DE (1993) An Introduction to Random Vibrations, Spectral and Wavelet Analysis. University of Cambridge, Longman, London: 295-370.

6. Chui CK, Montefusco L, Puccio L (1994) Wavelets: Theory, Algorithms and applications. Academic Press, San Diego.

7. Byrnes JS, Byrnes JL, Hargreaves KA, Berry K (1994) Wavelet and Their Application. Kluwer Academic Publishers, Netherlands.

8. Strang G, Nguyen T (1996) Wavelets and Filter Banks, Wellesley-Cambridge Press, MA: 72

9. Palavajjhala S, Motard RL, Joseph B (1994) In: Motard RL, Joseph B (eds)
Wavelet application in Chemical Engineering. Kluwer Academic Publishers, MA: 33-83.

10. Taswell C (1995) In: A. Antoniadis, G. Oppenheim (eds) Wavelet and Statistics. Springer-Verlag New York, New York: 361-375.

11. Buckheit JB, Donoho DL (1995) In: Antoniadis A, Oppenheim G (Eds) Wavelet and Statistics. Springer-Verlag New York, New York: 55-81.

12. Misiti M, Misiti Y, Oppenheim G, Poggi JM, Antoniadis A (1995) Wavelet and Statistics. Springer-Verlag New York, New York: 239-259.

13. Chui CK (1997) Wavelet: A Mathematical Tool for Signal Analysis. SIAM, Philadelphia.

14. Hubbard BB (1996) The World According to Wavelets: The Story of a Mathematical Technique in the Making. A.K. Peters, Wellesley.

15. Zou XY, Mo JY (1996) Spline-Wavelets Analysis of Step Voltammetry Signals Chem. J. Chin. Univ. 17: 1522-1527.

16. Fang H, Chen HY (1997) Anal. Chim. Acta 346: 319-325.

17. Bao LJ, Mo JY, Tang ZY (1997) The Application in Processing AnalyticalChemistry Signals of a Cardinal Spline Approach to Wavelets. Anal Chem 69: 3053-3057.

18. Zou XY, Mo JY (1997) Anal Chim Acta 340: 115-121.

19. Zou XY, Mo JY (1997) Chin Sci Bull 42(4): 382-385.

20. Zou XY, Mo JY (1997) Chin Sci Bull 42(8): 640-644.

21. Cody MA (1994) Dr. Dobbs's J 194: 44-54, 100.

22. Coifman RR, Meyer Y, Quake S, Wickerhauser MV, Byrnes JS (1994) Wavelet and Their Application. Kluwer Academic Publishers, Netherlands: 363-379.

23. Walczak B, Massart DL (1997) Chemom Intell Lab Syst 38: 39-50.

24. Walczak B, Massart DL (1997) Chemom Intell Lab Sys 36: 81-94.

25. Walczak B, Massart DL (1997) Trends Anal Chem 16: 451-463.

26. Alsberg BK, Woodward AM, Kell DB, Chemom Intell Lab Syst 37: 215-239.

27. Wang H, Xiao JH, Pan ZX, Zhang MS (1997) Chemistry Huaxue Tongbao 6: 20-23.

28. Depczynski U, Jetter K, Molt K, Niemoller A (1997) Chem In tell Lab Syst 39 19-27.

29. Brown S, Blank TB, Sum ST, Weyer LG (1994) Anal Chem 66: 315R-359R.

30. Brown S, Sum ST, Despagne F (1996) Anal Chem 68: 21R-62R.

31. Donoho DL (1993) In: Mayer Y, Roques S (Eds) Progress in Wavelet Analysis and Applications. Editions Frontiers, France: 109-128.

32. Donoho DL (1995) De-noising by soft-thresholding. IEEE Trans Inf Theory 41 613-627.

33. Permann DNS, Teitelbaum H (1993) J Phys Chem 97: 12670-12673.

34. Mittermayr CR, Nikolov SG, Hutter H, Grasserbauer M (1996) Chemom Intell Lab Syst 34: 187-202.

35. Barclay VJ, Bonner RF, Hamilton IP (1997) Anal Chem 69: 78-90.

36. Bush CA (1974) Anal Chem 46: 890-895.

37. Binkley DP, Dessy RE (1980) Anal Chem 52: 1335-1344.

38. Chau FT, Tam KY (1994) Comput Chem 18: 13-20.

39. Chau FT, Shih TM, Gao JB, Chan CK (1996) Application of the Fast Wavelet Transform Method to Compress Ultraviolet-Visible Spectra. Appl. Spectrosc. 50: 339-349.

40. Chau FT, Gao JB, Shih TM (1997) J Wang Appl Spectrosc 51: 649-659.

41. Leung KM, Chau FT (1997) Acta Phys Chim Sin 13: 857-864.

42. Thakur AN, Arnold JR (1993) Nucl Instrum Methods 325: 529-536.

43. Press WH, Teukolsky SA, Vetterling WT, Flannery BP (1992) Numerical Recipes in Fortran. Cambridge University Press, Cambridge: 490-602.

44. Bos M, Hoogendam E (1992) Anal Chim Acta 267: 73-80. 
Citation: Medhat ME (2015) A Review on Applications of the Wavelet Transform Technique in Spectral Analysis. J Appl Computat Math 4: 224. doi:10.4172/2168-9679.1000224

Page 6 of 6

45. Pan ZX, Shao XG, Zheng HB, Liu W, Wang H et al (1996) Chin J Anal Chem 24: $149-153$.

46. Shao XG, Cai WS, Pan ZX, Zhang MS (1997) In: Liang YZ, Nortvedt R, Kvalheim OM, Shen HL, Xu QS (Eds). Changsha: 205-206.

47. Shao XG, Cai WS, Sun PY, Zhang MS, Zhao GW (1997) Anal Chem 69: 17251725.

48. Shao XG, Sun PY, Cai WS, Zhang MS (1997) Chemistry Huaxue Tongbao 8: 59-62.

49. Shao XG, Sun PY, Cai WS, Zhang MS (1997) Chin J Anal Chem 25: 671-674.

50. Maeder M, Zuberbuehler AD (1986) Anal Chim Acta 181: 287-291.

51. Maeder M (1987) Anal Chem 59: 527-530.

52. Schostack KJ, Malinowski ER (1993) Chemom Intell Lab Syst 20: 173-182.

53. Kvalheim OM, Liang YZ (1992) Anal Chem 64: 936-953

54. Liang YZ, Kvalheim OM (1993) J Chemom 7: 15-43.

55. Mittermayr CR, Frischenschlager H, Rosenberg E, Grasserbauer M (1997) Filtering and integration of chromatographic data: a tool to improve calibration. Fresenius J Anal Chem 358: 456-464.

56. Mittermayr CR (1996) The Application of the Wavelet Transform to Chromatographic Data Processing, Dissertation, Vienna University for Technology, Austria.

57. Shen HL, Wang JH, Liang YZ, Chen WC (1997) Chem J Chin Univ 18: 530-534.

58. Shen HL, Wang JH, Liang YZ, Pettersson K, Josefson M, et al (1997) Chemom Intell Lab Syst 37: 261-269.

59. Maeder M, Zilian A (1988) Chemom Intell Lab Syst 3: 205-213.

60. Gemperline PJ (1986) Anal Chem 58: 2656-2663.

61. Stark PB, Herron MM, Matteson A (1993) Appl Spectrosc 47: 1820-1829.

62. Bos M, Vrielink JAM (1994) Chemom Intell Lab Syst 23: 115-122.

63. Walczak B, Bogert B, Massart DL (1996) Anal Chem 68: 1742-1747.
64. Alsberg BK, Woodward AM, Winson MK, Rowland J, Kell DB (1997) Analyst 122: 645-652.

65. Shew SL (1995) Method and Apparatus for Determining Relative Ion Abundances in Mass Spectrometry Utilizing Wavelet Transforms. US Patent 5,436,447, U.S Patent and Trademark Office, Washington, DC.

66. Nikolov SG, Hutter H, Grasserbauer M, Chemom Intell Lab Syst 34: 263-273.

67. Hutter H, Brunner C, Nikolov S, Mittermayr CR, Grasserbauer M (1996) Fresenius J Anal Chem 355: 585-590.

68. Wolkenstein M, Hutter H, Grasserbauer M (1997) Wavelet filtering for analytical data. Fresenius J Anal Chem 358: 165-169.

69. Wolkenstein M, Hutter H, Nikolov SG, Grasserbauer M (1997) Fresenius J Ana Chem 357: 783-788.

70. Strobel HA, Heineman WR (1989) Chemical Instrumentation: A Systematic Approach ( $3^{\text {rd }}$ edn). Wiley, New York 824-829: 1037-1039.

71. Hoch JC, Stern AS (1996) NMR Data Processing. Wiley-Liss, New York: 144 151

72. Guillemain P, Kronland-Martinet R, Martens B (1992) In: Meyer Y. (Ed) Wavelets and application: Proceedings of the Second International Conference on Wavelets and Their Applications, Marseille, France, May 1989. Springer Verlag, Paris: $38-60$.

73. Neue G (1996) Solid State. Nucl Magn Reson 5: 305-314.

74. Kemp W (1986) NMR in Chemistry: A Multinuclear Introduction. Macmillan London: 158-168.

75. Perkampus HH (1992) UV-VIS Spectroscopy and Its Applications. SpringerVerlag, Berlin: 26.

76. Gao JB, Chau FT, Shih TM (1996) SEA Bull Math 20: 85-90.

77. Liu W, Xiong JH, Wang H, Wang YM (1997) Chem J Chin Univ 6: 860-863.

78. Yan L, Mo JY (1995) Chin Sci Bull 40(17): 1567-1570.

79. 79. Chen J, Zhong HB, Pan ZX, Zhang MS (1996) Chin J Anal Chem 24: 1002 1006. 